\title{
ISOMETRIC IMMERSIONS WITH CONGRUENT GAUSS MAPS
}

\author{
JOHN DOUGLAS MOORE AND MARIA-HELENA NORONHA
}

(Communicated by Jonathan M. Rosenberg)

\begin{abstract}
A characterization of pairs of isometric immersions which have congruent Gauss maps is given.
\end{abstract}

\section{INTRODUCTION}

The purpose of this article is to present a refinement of some aspects of a beautiful theorem due to Dajczer and Gromoll [DG], which characterizes pairs of isometric immersions from a given Riemannian manifold into Euclidean space with congruent Gauss maps. We will reformulate part of the theory of circular isometric immersions, which is the proper context for the theorem, by systematically utilizing the notion of complexification.

The simplest case of two distinct isometric immersions with congruent Gauss maps is provided by the helicoid and the simply connected cover of the catenoid. These are conjugate minimal surfaces, i.e. the real and imaginary parts of a holomorphic map from a Riemann surface into $\mathbb{C}^{3}$, and they fit into a oneparameter family of associated minimal surfaces, which are pictured in do Carmo's text [dC, pp. 223-224]. All of the associated minimal surfaces have congruent Gauss maps.

More generally, it was discovered by Dajczer, Gromoll, and Rodriguez that a minimal isometric immersion $f$ from a simply connected Kähler manifold $M$ into a real Euclidean space $\mathbb{R}^{N}$ is the real part of a holomorphic map $h$ from $M$ into $\mathbb{C}^{N}$. The maps $f_{\theta}=r e\left(e^{i \theta} h\right)$, for $\theta \in \mathbb{R}$, are minimal isometric immersions with congruent Gauss maps, and the collection $\left\{f_{\theta}: \theta \in \mathbb{R}\right\}$ is called an associated family of minimal isometric immersions.

Theorem. Let $M$ be a complete simply connected Riemannian manifold, and suppose that

$$
f, \tilde{f}: M \longrightarrow \mathbb{R}^{N}
$$

Received by the editors October 26, 1988 and, in revised form, November 17, 1988.

1980 Mathematics Subject Classification (1985 Revision). Primary 53C42.

Key words and phrases. Minimal isometric immersion, Gauss map.

The first author was partially supported by NSF grant DMS 8700976 . The second author was partially supported by FAPESP, Sao Paulo, Brasil. 
are two isometric immersions with congruent Gauss maps. Then $M, f$, and $\tilde{f}$ have Riemannian product decompositions

$$
\begin{aligned}
& M=M_{0} \times M_{1} \times \cdots \times M_{k}, \\
& f=f_{0} \times f_{1} \times \cdots \times f_{k}: M_{0} \times M_{1} \times \cdots \times M_{k} \longrightarrow \mathbb{R}^{N_{0}} \times \mathbb{R}^{N_{1}} \times \cdots \times \mathbb{R}^{N_{k}}, \\
& \tilde{f}=\tilde{f}_{0} \times \tilde{f}_{1} \times \cdots \times \tilde{f}_{k}: M_{0} \times M_{1} \times \cdots \times M_{k} \longrightarrow \mathbb{R}^{N_{0}} \times \mathbb{R}^{N_{1}} \times \cdots \times \mathbb{R}^{N_{k}},
\end{aligned}
$$

where $N=N_{0}+N_{1}+\cdots+N_{k}$, and each $M_{i}$, for $1 \leq i \leq k$, possesses a complex structure making it into a Kähler manifold, so that

(1) $f_{0}$ and $\tilde{f}_{0}$ differ by a rigid motion, and

(2) for $1 \leq i \leq k, f_{i}$ and $\tilde{f}_{i}$ are members of an associated family.

\section{CirCUlar ISOMETRIC IMMERSIONS}

Minimal isometric immersions of Kähler manifolds into real Euclidean spaces are orthogonal projections of holomorphic maps. To see this, recall that the complexified tangent bundle of a Kähler manifold $M^{2 n}$ of real dimension $2 n$ divides into a Whitney sum,

$$
T M \otimes \mathbb{C}=T_{1,0} M \oplus T_{0,1} M,
$$

with $T_{1,0} M$ denoting the holomorphic tangent bundle, $T_{0,1} M$ the image of $T_{1,0} M$ under the conjugation operation $z \mapsto \bar{z}$. The curvature operator $R$ : $\Lambda^{2} T M_{p} \rightarrow \Lambda^{2} T M_{p}$ extends to a complex linear map

$$
R: \Lambda^{2} T M_{p} \otimes \mathbb{C} \rightarrow \Lambda^{2} T M_{p} \otimes \mathbb{C},
$$

which satisfies

$$
R(z \wedge w)=R(\bar{z} \wedge \bar{w})=0
$$

for all $z, w \in T_{1,0} M_{p}$.

Suppose now that $f: M^{2 n} \rightarrow \mathbb{R}^{N}$ is an isometric immersion. We extend the Riemannian metric $\langle$,$\rangle of M$ and the second fundamental form $\alpha$ of $f$ to complex bilinear maps

$$
\begin{aligned}
\langle,\rangle & :\left(T M_{p} \otimes \mathbb{C}\right) \times\left(T M_{p} \otimes \mathbb{C}\right) \longrightarrow \mathbb{C}, \\
\alpha & :\left(T M_{p} \otimes \mathbb{C}\right) \times\left(T M_{p} \otimes \mathbb{C}\right) \longrightarrow\left(N M_{p} \otimes \mathbb{C}\right),
\end{aligned}
$$

which satisfy the complex Gauss equations,

$$
\langle R(x \wedge y), z \wedge w\rangle=\langle\alpha(x, z), \alpha(y, w)\rangle-\langle\alpha(x, w), \alpha(y, z)\rangle,
$$

for $x, y, z, w \in T M_{p} \otimes \mathbb{C}$.

Definition. The isometric immersion $f: M^{2 n} \rightarrow \mathbb{R}^{N}$ is circular if $\alpha(z, \bar{w})=0$, for all $z \in T_{1,0} M_{p}, \bar{w} \in T_{0,1} M_{p}$.

Equivalently, $f$ is circular if its real-valued second fundamental form satisfies

$$
\alpha(J x, J y)+\alpha(x, y)=0
$$


for $x, y \in T M_{p}$, where $J$ is the almost complex structure. It follows from this characterization that a circular isometric immersion is automatically minimal. Dajczer and Rodriguez [DR, Theorem 1.2] prove the converse: a minimal isometric immersion from a Kähler manifold into a real Euclidean space is necessarily circular. Indeed, if $\left(e_{1}, \ldots, e_{n}\right)$ is a unitary basis for $T_{1,0} M_{p}$, then minimality implies (via a straightforward calculation) that

$$
\sum_{i=1}^{n} \alpha\left(e_{i}, \bar{e}_{i}\right)=0
$$

Hence if $z \in T_{1,0} M_{p}$, it follows from the Gauss equations that

$$
\begin{aligned}
0 & =\sum_{i=1}^{n}\left\langle R\left(z \wedge e_{i}\right), \bar{z} \wedge \bar{e}_{i}\right\rangle \\
& =\sum_{i=1}^{n}\left[\left\langle\alpha(z, \bar{z}), \alpha\left(e_{i}, \bar{e}_{i}\right)\right\rangle-\left\langle\alpha\left(z, \bar{e}_{i}\right), \alpha\left(\bar{z}, e_{i}\right)\right\rangle\right. \\
& =-\sum_{i=1}^{n}\left\|\alpha\left(z, \bar{e}_{i}\right)\right\|^{2} .
\end{aligned}
$$

It follows immediately that $\alpha\left(z, \bar{e}_{i}\right)=0$ for all $i$, which is exactly the circular condition, since $z \in T_{1,0} M_{p}$ was chosen arbitrarily.

Suppose now that $f: M \rightarrow \mathbb{R}^{N}$ is a circular isometric immersion from a simply connected Kähler manifold $M$ with second fundamental form $\alpha$. We can define

$$
\tilde{\alpha}: T M_{p} \times T M_{p} \longrightarrow N M_{p}
$$

by $\tilde{\alpha}(x, y)=-\alpha(x, J y)$. Leaving the connections on $T M$ and $N M$ unchanged, we can replace the second fundamental form $\alpha$ by $\tilde{\alpha}$, and the new data still satisfy the Gauss, Codazzi, and Ricci equations. Hence it follows from the fundamental existence and uniqueness theorem [BC, Chapter 10, Theorem 5] that there is an isometric immersion $\tilde{f}: M \rightarrow R^{N}$, unique up to a rigid motion, which induces the new data. Moreover,

$$
h=f+i \tilde{f}: M^{2 n} \longrightarrow \mathbb{C}^{N}=\mathbb{R}^{N}+i \mathbb{R}^{N}
$$

is a holomorphic map, isometric after the Kähler metric of $M^{2 n}$ is multiplied by $\sqrt{2}$. The given circular immersion lies in an associated family $\left\{f_{\theta}=\operatorname{Re}\left(e^{i \theta} h\right)\right.$ : $\theta \in \mathbb{R}\}$; all the members of the associated family have the same tangent and normal connection, and their second fundamental forms satisfy the identity

$$
\alpha_{\theta}(z, w)=e^{i \theta} \alpha(z, w), \quad \text { for } z, w \in T_{1,0} M_{p} .
$$

It now follows from Calabi's rigidity theorem $[C]$ that any two minimal isometric immersions from the same simply connected Kähler manifold $M^{2 n}$ into Euclidean space $\mathbb{R}^{N}$ must be orthogonal projections of the same holomorphic 
map from $M^{2 n}$ into $\mathbb{C}^{N}$. The moduli space of such minimal isometric immersions is a linear variety in the closure of a Siegel domain, as described in $[\mathrm{L}$, Chapter IV, Theorem 9].

\section{PROOF OF THE THEOREM}

Suppose that $M$ is a complete simply connected Riemannian manifold, and that $f, \tilde{f}: M \longrightarrow \mathbb{R}^{N}$ are two isometric immersions with congruent Gauss maps. This means that after subjecting $\tilde{f}$ to a rigid motion, we can construct an orthogonal linear transformation

such that

$$
\phi_{p}: T M_{p} \longrightarrow T M_{p}
$$

$$
d f_{p} \circ \phi_{p}=d \tilde{f}_{p}: T M_{p} \longrightarrow \mathbb{R}^{N},
$$

for each $p \in M$. The $\phi_{p}$ 's fit together to form a vector bundle map $\phi: T M \rightarrow$ $T M$. If $X$ is a section of $T M$,

$$
X(\tilde{f})=d \tilde{f}(X)=d f(\phi(X))=\phi(X)(f) .
$$

Note that since $d f_{p}\left(T M_{p}\right)=d \tilde{f}_{p}\left(T M_{p}\right)$, the normal bundles of $f$ and $\tilde{f}$ are the same and have the same normal connections. Denoting the second fundamental forms of $f, \tilde{f}$ by

we claim that

$$
\alpha, \tilde{\alpha}: T M_{p} \times T M_{p} \longrightarrow N M_{p},
$$

$$
\alpha\left(\phi_{p} x, y\right)=\tilde{\alpha}(x, y)
$$

for $x, y \in T M_{p}$. Indeed, if $\mathrm{X}$ is a vector field extension of $x$ and $n \in N M_{p}$,

$$
\begin{aligned}
n \cdot \alpha\left(\phi_{p} x, y\right) & =n \cdot\left(d^{2} f\right)_{p}\left(y, \phi_{p}(x)\right)=n \cdot y(\phi(X)(f)) \\
& =n \cdot y(X(\tilde{f}))=n \cdot d^{2} \tilde{f}(x, y)=n \cdot \tilde{\alpha}(x, y) .
\end{aligned}
$$

Lemma 1. If $\nabla$ is the Levi-Civita connection on $M$, then $\left[\nabla_{x}, \phi\right]=0$, for $x \in T M_{p}$, i.e. $\phi$ is a parallel section of $\operatorname{Hom}(T M, T M)$ with respect to $\nabla$.

Proof. The Levi-Civita connection $\nabla$ is the pullback via either $f$ or $\tilde{f}$ of the tangential component of the Euclidean conection on $\mathbb{R}^{N}$. Since $d \tilde{f}=d f \circ \phi$, the Levi-Civita connection is invariant under pullback via $\phi$, i.e. $\left[\nabla_{x}, \phi\right]=0$.

Now we extend $\phi$ to a complex linear endomorphism of $T M \otimes \mathbb{C}$. Since $\phi$ is orthogonal, its eigenvalues $\lambda$ are of length one. It follows from Lemma 1 that

$$
E_{\lambda}=\{v \in T M \otimes \mathbb{C}: \phi(v)=\lambda v\}
$$

is a parallel distribution on $M$. Thus we have a direct sum decomposition into parallel distributions

$$
T M \otimes \mathbb{C}=E_{1} \oplus E_{-1} \oplus \sum_{i=1}^{k}\left(E_{\lambda_{i}} \oplus E_{\bar{\lambda}_{i}}\right),
$$

where $\lambda_{i}, 1 \leq i \leq k$, are the distinct eigenvalues of the form $e^{i \theta}$, for $0<\theta<\pi$. 
Lemma 2. If $\langle$,$\rangle and \alpha$ are extended complex linearly to $T M_{p} \otimes \mathbb{C}$,

(1) $\left\langle E_{\lambda}, E_{\mu}\right\rangle=0$ unless $\lambda=\bar{\mu}=1 / \mu$,

(2) $\alpha\left(E_{\lambda}, E_{\mu}\right)=0$ unless $\lambda=\mu$.

Proof. If $x \in E_{\lambda}, y \in E_{\mu}$,

$$
\lambda\langle x, y\rangle=\langle\phi x, y\rangle=\left\langle x, \phi^{-1} y\right\rangle=(1 / \mu)\langle x, y\rangle \Rightarrow(\lambda-(1 / \mu)\langle x, y\rangle=0,
$$

so either $\langle x, y\rangle=0$ or $\lambda=1 / \mu$. Similarly,

$$
\begin{aligned}
\lambda \alpha(x, y) & =\alpha(\phi x, y)=\tilde{\alpha}(x, y)=\alpha(x, \phi y)=\mu \alpha(x, y) \\
& \Rightarrow(\lambda-\mu) \alpha(x, y)=0,
\end{aligned}
$$

so either $\alpha(x, y)=0$ or $\lambda=\mu$.

Lemma 3. $M$ divides into a Riemannian product,

$$
M=M_{1} \times M_{-1} \times \prod_{i=1}^{k} M_{\lambda_{i}},
$$

where $T M_{1}=E_{1}, T M_{-1}=E_{-1}$, and $T M_{\lambda_{i}}=E_{\lambda_{i}} \oplus \bar{E}_{\lambda_{i}}$.

Proof. This is a direct consequence of the de Rham decomposition theorem [KN, Chapter 4, Theorem 6.1], since the holonomy at each point divides into a direct sum.

Lemma 4. The isometric immersions $f$ and $\tilde{f}$ divide into Riemannian products

$$
f=f_{1} \times f_{-1} \times f_{\lambda_{1}} \times \cdots \times f_{\lambda_{k}}, \quad \tilde{f}=\tilde{f}_{1} \times \tilde{f}_{-1} \times \tilde{f}_{\lambda_{1}} \times \cdots \times \tilde{f}_{\lambda_{k}}
$$

where $f_{1}, \tilde{f}_{1}: M_{1} \rightarrow \mathbb{R}^{N_{1}}, \ldots, f_{\lambda_{k}}, \tilde{f}_{\lambda_{k}}: M_{\lambda_{k}} \rightarrow \mathbb{R}^{N_{\lambda_{k}}}$.

Proof. This is a direct consequence of the Main Lemma of [M], together with Lemmas 2 and 3.

It remains now to investigate the individual factors. Let $M_{r}=M_{1} \times M_{-1}$, $f_{r}=f_{1} \times f_{-1}, \tilde{f}_{r}=\tilde{f}_{1} \times \tilde{f}_{-1}$.

Lemma 5. $f_{r}$ and $\tilde{f}_{r}$ differ by a rigid motion.

Proof. $f_{1}$ and $\tilde{f}_{1}$ differ by a rigid motion by the uniqueness part of the fundamental existence and uniqueness theorem [BC, loc. cit.], because $f_{1}$ and $\tilde{f}_{1}$ have isomorphic tangent and normal connections, and isomorphic second fundamental forms.

The second fundamental forms $\alpha_{-1}, \tilde{\alpha}_{-1}$ of $f_{-1}, \tilde{f}_{-1}$ satisfy the relation $\alpha_{-1}=-\tilde{\alpha}_{-1}$, but after an isometry of normal bundles $(n \mapsto-n)$, we again have the same initial data for the fundamental existence and uniqueness theorem, so $f_{-1}$ and $\tilde{f}_{-1}$ also differ by a rigid motion.

We now focus on $f_{\lambda_{i}}, \tilde{f}_{\lambda_{i}}: M_{\lambda_{i}} \rightarrow \mathbb{R}^{N_{\lambda_{i}}}$. Let

$$
J: T M_{\lambda_{i}} \otimes \mathbb{C} \longrightarrow T M_{\lambda_{i}} \otimes \mathbb{C}
$$


by

$$
J(z)=\left\{\begin{aligned}
i z, & \text { for } z \in E_{\lambda_{i}} \\
-i z, & \text { for } z \in \bar{E}_{\lambda_{i}} .
\end{aligned}\right.
$$

Then $J^{2}=-I$ and $J$ is real, i.e. $J(\bar{z})=\overline{J(z)}$. Moreover, $J$ is parallel by construction.

Lemma 6. $J$ makes $M_{\lambda_{i}}$ into a Kähler manifold with holomorphic tangent space $T_{1,0} M_{\lambda_{i}}=E_{\lambda_{i}}$.

Proof. Clearly $J$ is Hermitian,

$$
\langle J x, J y\rangle=\langle x, y\rangle,
$$

for $x, y \in T M_{\lambda_{i}} \otimes \mathbb{C}$, and is preserved by the Levi-Civita connection $\nabla$. The integrability of $J$ follows from the integrability of $T_{1,0} M=E_{\lambda_{i}}$, which in turn, follows from the fact that $\nabla$ preserves $E_{\lambda_{i}}$ :

$$
X, Y \in \Gamma\left(E_{\lambda_{i}}\right) \Rightarrow[X, Y]=\nabla_{X} Y-\nabla_{Y} X \in \Gamma\left(E_{\lambda_{i}}\right) \text {. }
$$

Lemma 7. $f_{\lambda_{i}}$ and $\tilde{f}_{\lambda_{i}}$ are circular.

Proof. It follows from Lemma 2 that the second fundamental forms $\alpha_{\lambda_{i}}, \tilde{\alpha}_{\lambda-i}$ of $f_{\lambda_{i}}, \tilde{f}_{\lambda_{i}}$ satisfy

$$
\alpha_{\lambda_{i}}(x, \bar{y})=0, \quad \tilde{\alpha}_{\lambda_{i}}(x, \bar{y})=0,
$$

for $x \in E_{\lambda_{i}}=T_{1,0} M_{\lambda_{i}}, \bar{y} \in T_{0,1} M_{\lambda_{i}}$.

$$
\begin{aligned}
& \text { If } z, w \in T_{1,0} M_{\lambda_{i}}, \\
& \qquad \tilde{\alpha}_{\lambda_{i}}(z, w)=\alpha_{\lambda_{i}}(\phi z, w)=\lambda_{i} \alpha(z, w),
\end{aligned}
$$

and hence by the discussion in $\S 2, f_{\lambda_{i}}$ and $\tilde{f}_{\lambda_{i}}$ have the second fundamental forms of associated circular immersions. Since the connections in tangent and normal bundles are the same, it follows from the fundamental existence and uniqueness theorem that $f_{\lambda_{i}}$ and $\tilde{f}_{\lambda_{i}}$ are associated isometric immersions. This finishes the proof of the theorem.

\section{REFERENCES}

[BC] R. L. Bishop and R. J. Crittenden, Geometry of manifolds, Academic Press, New York, 1964.

[C] E. Calabi, Isometric imbeddings of complex manifolds, Ann. Math. 58 (1953), 1-23.

[DG] M. Dajczer and D. Gromoll, Real Kähler submanifolds and the uniqueness of the Gauss map, J. Differential Geom. 22 (1985), 13-28.

[DR] M. Dajczer and L. Rodriguez, Rigidity of real Kähler submanifolds, Duke Math. J. 53 (1986), 211-220.

[dC] M. do Carmo, Differential geometry of curves and surfaces, Prentice-Hall, Englewood Cliffs, NJ, 1976.

[KN] S. Kobayashi and K. Nomizu, Foundations of differential geometry, vol. 1, Wiley, New York, 1963. 
[L] H. B. Lawson, Lectures on minimal submanifolds, Publish or Perish, Berkeley, CA, 1980.

[M] J. D. Moore, Isometric immersions of Riemannian products, J. Differential Geom. 5 (1971), 159-168.

Department of Mathematics, University of California, Santa Barbara, California 93106

Departamento de Matematica IMECC, UniCamp CP 6165, 13083 Campinas Sao Paulo, BRASIL 